\title{
Automatic Parking Monitoring System using IOT
}

\author{
Er. Bharti Sood1, Er. Rahul Gupta² \\ ${ }^{1}$ Electronics \& Communications Engineering, ${ }^{2}$ Electrical and Electronics Engineering, \\ Maharaja Agrasen University Baddi, Himachal Pradesh, India
}

\begin{abstract}
With the increase in world population, vehicle production, giant industrialization and slow paced city development more and more parking spaces and facilities are required. With the advancement in technology now we can relate technology with our normal parking system resulting a development of a new era of parking system called as smart parking system. In this paper a new parking system called smart parking system to assist drivers to find vacant spaces in short time. This parking system works in two phases. In 1st phase the detection of vehicles in various parking slots using various sensors takes place, while the 2nd phase aimed at communicating the collected data to user remotely. The SPS provide various advantages including vacant parking space detection, improper parking detection, payment methods
\end{abstract}

\section{KEYWORDS: Ultrasonic Sensor, Arduino Board, SPS}

\section{INTRODUCTION}

Now a days searching for vacant parking slots in metropolitan area is the issue for most drivers, and it is very time consuming. Just cruising for vacant parking slots results more Traffic congestion and air pollution[1]. According to the survey of the metropolitan city about $40 \%$ of the total traffic is generated by vehicles searching for the vacant parking spaces. To avoid this traffic congestion problems and improve the convenience for drivers many smart parking are introduced in order to avoid traffic congestion and also provide a better convenient parking system for public. In traditional parking system there is no guarantee of finding available parking space.In this Project, we mainly focus on designing a new smartparking system that assists drivers to find parking spaces in aspecific parking district. In addition, an important goal of the system is to reduce the traffic searching for parking, hencereduce energy consumption and air pollution.For instance, in Malaysia window-shopping, or visiting shopping complexes simply for looking rather than buying, is a common activity[2].

With the rapid development in each and every sector of society that can be hospitals, transportation, shopping complexes etc. providing sufficient, secure parking facilities is one of the main issues in developing sectors if the society. In this paper we are introducing a smart parking system (SPS) which is very cheap, convenient, and easy to use which provide better facility to drivers. This paper is organized as follows: the introduction details the parking lots. Part 2 discusses current parking lot problems and the difficulties that customers encounter in parking lots. Section 3 gives a system overview and the features of SPS. Section 4 outlines SPS architecture and the devices required to implement it. Finally, the last section offers conclusions and discusses current research.

\section{Problem Regarding Parking System:}

Difficulty in finding vacant parking slots: With the rapid growth in population anf vehicles production, finding a vacant space in any metropolitan city is difficult, but it gets very hard on weekends. A shows that $86 \%$ of drivers face difficulty in finding a parking space in multilevel parking lots [3]. Stadiums and shopping complexes are crowded at peak periods and difficulty in finding vacant slots at these places is a major problems for customers [4]. Insufficient car parks spaces leads to the traffic congestion and driver frustration [5].

\section{Improper Parking:}

Parking slots are designed in such a way that any vehicle respective of their shape and size can be parked in provided parking spaces. Improper parking can happen when the driver parks the vehicle in improper way or a bit outside the parking space this causes trouble to other people.

\section{Parking Fee Management:}

Different types of methods are currently being used for parking payment. There are many technologies for facilitating parking payments, whether via a cashier, or a fully automated self-serve system. But all these payment methods are time consuming. Parking fee payment methods plays an important role in traffic congestion problem. When the payment method is fast \&convenient there will be less congestion of vehicles and vice versa.

\section{A. Smart Parking System Description:}

This system is using the ultrasonic sensor as detector to detect the car park availability. The project also as known as Smart Parking System contain few features such as vacant car park detection, improper parking detection, display available parking lot and directional indicators towards the vacant car park space, payment facilities and different types of parking spaces by using LED indicator. In every parking slot, a set of Ultra Sonic sensors have to be installed. These sensors would monitor the real-time data about the availability of slots to nearby drivers. Changes in the availability of parking slots will be reflected through a status change of the sensors and that, in turn, will be collected in a gateway. Unlike the sensors, the gateways have to be operational round-the-clock. The data collected in the gateway are then sent on to the centralized server.

Traffic congestion caused by vehicles is an alarming problem at a global scale and it has been growing exponentially. Car parking problem is a major contributor and has been still a major problem with confined parking spaces in urban cities. Searching for a parking space is a routine (and often 
frustrating) activity for many people in cities around the world. This search burns about one million barrels of the world's oil every day. These problems will get worse as the global population continues to urbanize without a wellplanned, convenience-driven retreat from the car. According to a report Smart Parking could result in saving 2, 20,000 gallons of fuels till 2030 and approx. 3, 00,000 gallons of fuels by 2050 if implemented successfully. Smart Parking systems obtain information about available parking spaces in a particular geographic area. This process is real-time to place vehicles at available positions. It involves real-time data collection using low-cost sensors and mobile-phoneenabled automated payment systems that allows people to reserve parking in advance.

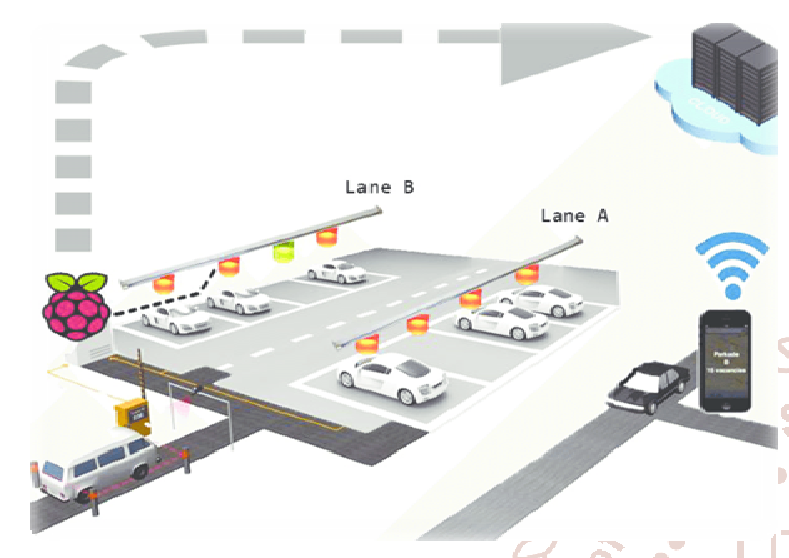

Figure 1: Proposed system

\section{Server:}

The server in a smart parking setup needs to have 2-way communication protocol support. On the one hand, it will receive the data from the gateways about the free and occupied parking slots in any facility. At the other end, it will send real-time notifications to the dedicated mobile app in the user's device - to guide them to the parking area, and the particular 'vacant' parking slot. A detailed map of the parking facility will also be sent from the server to the user, for additional guidance.

\section{Mobile application:}

The final component of the smart parking architecture is the dedicated mobile app which serves as the touch point for the final users. After receiving notifications from the server, the app would help the driver to navigate to the empty parking slot. For this navigation, the application will either rely on the tools placed in the parking slots or the phone GPS.

\section{Architecture Design:}

Ultrasonic sensor detects the vehicle - send yes signal to microcontroller - controller initiate vehicle presence command in data base.

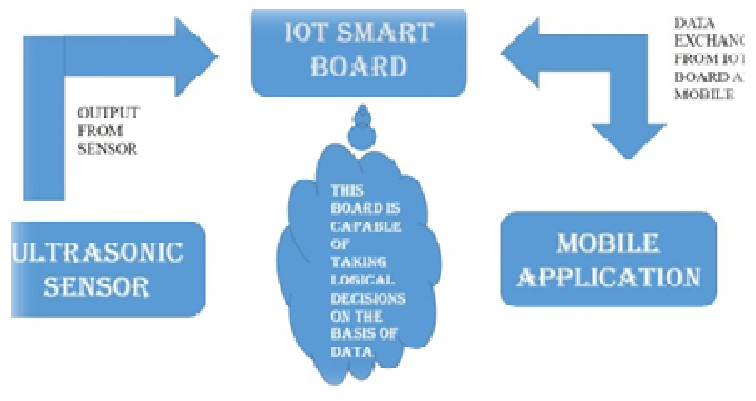

Figure 2: Working Model

If ultrasonic sensor detect no vehicle - send no signal to (0) controller- controller initiate empty command in data base. When digital signal initiate WI-FI. It connect with android platform and WI-FI send real time data string to application. System can detect status of parking. When system detects parking space from stored data base the user can easily access the easiest path for that position.

\section{Conclusion:}

The main motive of this study is to introduce parking system to avoid the problem i.e., finding an empty space and propose a solution. Ultrasonic sensors can be used both for parking space detection and improper parking detection. The proposed system for a parking detection system would decrease searching time for vacant spaces and reduce instances of single cars improperly parking across two spaces. Future research might examine car park booking procedures and optimization of sensor usage. Cost effectiveness and marketing could be studied as well.

\section{References}

[1] Ajzen, M. F. (1975). Belief, Attitude and behavior, and an Introduction to theory and beResearch. AddisonWesley.

[2] P.W.H. Coopers, "From Beijing to Budapest - Winning Brands. Winning Formats”, (2005), pp. 126.

[3] Kianpisheh, N. Mustaffa, J. M. Y. See and P. Keikhosrokiani, "User Behavioral Intention toward Using Smart Parking System", Proceeding of ICIEIS, (2011) Kuala Lumpur, Malaysia, pp. 732-747.

[4] D. B. L. Bong, K. C. Ting and K. C. Lai, "Integrated Approach in the Design of Car Park Occupancy Information System (COINS)", IAENG International Journal of Computer Science, IJCS, vol. 35, no. 1, (2008).

[5] M. Y. I. Idris, E. M. Tamil, N. M. Noor and K. W. Fong, "Parking Guidance System Utilizing Wireless Sensor Network and Ultrasonic Sensor", Information Technology Journal, ISSN 1812-5638, (2009). 